\title{
Central Monitor Based on Personal Computer Design with SpO2 and Body Temperature Parameters Using Wireless Xbee Pro
}

\author{
I Komang Yogi Mahardika1, Bambang Guruh Irianto1, Torib Hamzah¹, Shubhrojit Misra² \\ ${ }^{1}$ Department of Medical Electronics Engineering Technology Politeknik Kesehatan Kementerian Kesehatan Surabaya, Jl. Pucang Jajar Timur No. 10, Surabaya \\ (60245), Indonesia \\ ${ }^{2}$ Department of Electronics and Telecommunication Engineering, Jadavpur University, 188, Raja S.C. Mallick Rd, Kolkata, West Bengal 700032, India.
}

Corresponding Author: Bambang Guruh Irianto (bgi_ps@yahoo.com)

\begin{abstract}
Central patient monitor that is not realtime and continues will cause inaccuracies monitoring results and also sending data that is still using cable will cause limited distance. The purpose of this research is to design central monitoring based personal computer via Xbee Pro. The contribution of this research is the system works in realtime and continues, more parameters, using wireless, longer transmission distances. So that monitoring can be done in realtime and continue via Wireless with more distance, then the wireless system uses the Xbee Pro module, which has larger output power and uses the same number of wireless modules between transmitter and receiver. Body temperature was measured using the LM35 sensor, and oxygen saturation in the blood was measured using the MAX30100 sensor. Data is sent using Xbee Pro and displayed on a personal computer. At the distance of receiving data, approximately 25 meters with a wall divider, obtained results of smooth monitoring without any loss of data. The results showed that the average $\mathrm{SpO} 2$ error value was $0.34 \%$ in module 1 and $0.68 \%$ in module 2 . The average value of body temperature error was $0.46 \%$ in module 1 and $0.72 \%$ in module 2 . The results of this research can be implemented in a centralized patient monitoring system at the hospital, making it easier for health workers to monitor multiple patients, with the results of monitoring in realtime and continue more parameters via Wireless with greater distance.
\end{abstract}

INDEXT TERM Body Temperature, SpO2, Xbee Pro, Central Monitoring

\section{INTRODUCTION}

The proposed patient monitoring system will be beneficial for medical practitioners to carry out appropriate treatments and will also be beneficial for health care providers to improve disease management.[1]. Measuring and monitoring the bio-parameters of a patient such as ECG, RESP, SpO2, Blood pressure NIBP and temperature TEMP is an effective method to assist the doctor during diagnosis and treatment now[2]. The use of patient monitor equipment will be maximized if the monitoring is done centrally or centrally in one monitor screen. The centralized patient monitoring system plays an important role in the medical field where there are multiple physiological monitoring data parameters from several patients who support the central monitor to be seen by nurses[3]. One of the hospitals in Blitar, Java, Indonesia, namely Mardi Waluyo Regional Hospital, precisely in the infectious rose room installed a few centralized patient monitors, but still uses via cable as a link between the device and the monitoring so that it requires special installation which is a bit complicated for the wiring, especially the distance between the patient's place and the monitor room that requires precise measurement of cable length. Along with the development of technology at this time, it is possible to monitor health, such as using wireless networks or wireless systems that are connected between monitoring devices to the central monitor (PC), which is more practical than using cables. In recent years there have been rapid developments in wireless technology, where wireless networks have more applications than wire technology and are often used for wireless monitoring control applications in industrial and domestic environments that have longer lifespan and have lower data rates[4]. The development of wireless biomedical development measurements are the most

Homepage: http://jeeemi.org 
promising and is one that has the best application for the treatment of patients with chronic conditions[5]. The development of network and communication technology, the inconvenience of wiring is solved with the Wireless Sensor Network[6]. Monitoring the vital signs of people in hospitals (outpatients, emergency rooms, and post-operative care), patients diagnosed with chronic diseases using WSN technology becomes very important[7]. SpO2 or blood oxygen concentration in the blood, which is an important physiological parameter of the respiratory and circulatory system[8] when it decreased, causes the heart rate to become faster-experiencing fatigue, impaired vision, and dizziness, therefore checking for $\mathrm{SpO} 2$ is important in the world of health. Reading of $\mathrm{SpO} 2$ (peripheral oxygen saturation) is not always identical to the more desirable reading of $\mathrm{SaO} 2$ (arterial oxygen saturation) from arterial blood gas analysis; the two are correlated well enough that the safe, convenient, noninvasive, inexpensive pulse oximetry method is valuable for measuring oxygen saturation in clinical use[9]. Not only does $\mathrm{SpO} 2$ need to be monitored in patients, but the patient's body temperature is another parameter to help diagnose the condition of patients who have normal temperature ranges. Body temperature is a good indicator of one's health because most diseases are produced by body temperature or fever, and we can know these indicators about the health of the human body to achieve the goals and diagnosis for the appropriate disease [10]. Although the body temperature measured in individuals can vary in value, a healthy human body can maintain a consistent body temperature of around $37^{\circ} \mathrm{C}$. [11]. This monitoring system should reduce the heavy maintenance task of automatically monitoring the condition of the patient's body temperature in the hospital bed and can provide useful health information such as sleep structure analysis[12]. Compared with other mature short-distance wireless communications technologies, such as Bluetooth, WiFi, and so on, ZigBee consumes less power, has long-range \& low data rate, reliability, and security[13][14][15]. One of the wireless communication modules with ZigBee standard communication is XBee, produced by Digi International. XBee is a module that consists of an RF receiver and RF transmitter with a UART serial communication interface so that it is more easily integrated with a microcontroller. The transmission distances of ZigBee are about 10-1,500 meters, depending on power output, antenna, and environmental characteristics[16]. Xbee provides a wireless medium for interconnection and communication between devices, allowing the creation of pointto-multipoint networks and point-to-point networks[17]. With the central monitor via Wireless, nurses or doctors can monitor without having to repeatedly do data collection on each patient's bed but can be via a PC placed in the nurse station room.

Many researchers have made wireless systems that will later become the foundation in making a central monitor system. The research entitled monitoring heart rate and body temperature using wireless technology (Zigbee) in 2014[18], the study is to monitor BPM conditions and the patient's body temperature transmitted to the computer using the Xbee module. However, monitoring is only limited to BPM and body temperature, so that it is less optimal in terms of completeness of patient health parameters. The other study proposed the ZigBee-Based Multipoint to Point ECG Monitoring in 2014[19]. This device uses a central monitoring system, but monitoring is more devoted to ECG monitoring and will be more effective when monitoring is applied to several parameters so that it can monitor the patient's condition in more detail. Another research which using XBee wireless blood pressure monitoring system with Microsoft Visual Studio Computer Interfacing was proposed in 2015 [20]. The main concept of the project is to cancel out the cable connection between the computer and blood pressure sensor by using the XBee. However, if seen from the completeness of the parameters are still limited to blood pressure. Another research which based on wireless system is to monitor of temperature and humidity using sensor array in 2015 [21], aims to develop an array of sensors consisting of two signal conditioning circuits for capacitive humidity and resistive temperature sensors that are able to measure humidity and temperature wirelessly. However, monitoring for completeness of parameters is still limited to temperature and humidity, and the display is still using comport. Research in 2016 which proposed multiuser vital sign monitoring system using ZigBee wireless sensor network [22], data is transmitted using the ZigBee module, and signal parameters can be viewed from a computer wirelessly. However, monitoring uses two computers so that it cannot display central in one computer. Another research-based on heart rate monitoring system in 2017 [23], this device only sends ECG signals by telemetry, meaning that this device cannot be used to monitor SOP2, Respiration, and Temperature parameters. Next, research that used a computer system to monitor the heart rate was also proposed in 2017 [24]. This device uses Arduino as a data processor and is displayed on a PC, however sending data using RS232 serial or cable media.

Based on the weaknesses and limitations that have been mentioned in previous researchers among others, 1) Incomplete parameters, 2) Still using cable in data transmission, 3) Monitoring can not be in real-time and continue, 4) Short data transmission distance, so the purpose of this research is to be able to do monitoring through the central system using wireless or without using cable media as a delivery, monitoring can be done in real-time and continue with more complete parameters, and longer monitoring distances because Xbee has a greater transmit power output, so it has a range of operations at a greater distance than the wireless module in previous studies.

This article is composed of: Chapter II contains Material and Methods, Chapter III about the results of the research, Chapter IV Discussion, and Chapter V Conclusion.

\section{MATERIALS AND METHODS}

\section{A. EXPERIMENTAL SETUP}

This study was applied to patients who were taken data 6 times with a time interval of 1 minute. Data is taken in realtime and continues between module 1 and module 2. Data collection is done at a distance of $8,10,25,30$ with a wall barrier. Data 
collection was performed with 2 patients simultaneously. Parameters used by $\mathrm{SpO} 2$ and Body Temperature

\section{B. MATERIALS AND DEVICE}

This study uses the MAX30100 sensor to monitor oxygen saturation in the blood $(\mathrm{SpO} 2)$ and 2 resistors are used to pull up before entering the microcontroller. LM35 waterproof sensor was used to monitor body temperature. The minimum system ATMega328 was used to process data from sensors and Xbee Pro wireless module as data transmission. The Delphi 7.0 application is used to display centralized monitoring on personal computers. Finger Pulse Oximeter with merk Elitech Technovision type of FOX 1 and Digital Thermometer with merk Magic Star type of MS-202 were used as a means of comparison.

\section{EXPERIMENT}

In this research. There are 2 devices which will send data of $\mathrm{SpO} 2$ and TEMP using 2 Xbee Pro wireless module, then the data is received with 2 Xbee Pro wireless module which will be connected together to a personal computer using a USB HUB and displaying data in the form of digital numbers on a personal computer. The researcher conducted several tests including:

a. Comparison of module measurement results used for $\mathrm{SpO} 2$ using a pulse oximeter and Body Temperature using digital thermometer

b. Displays central with distance of $8,10,25,30$ meters

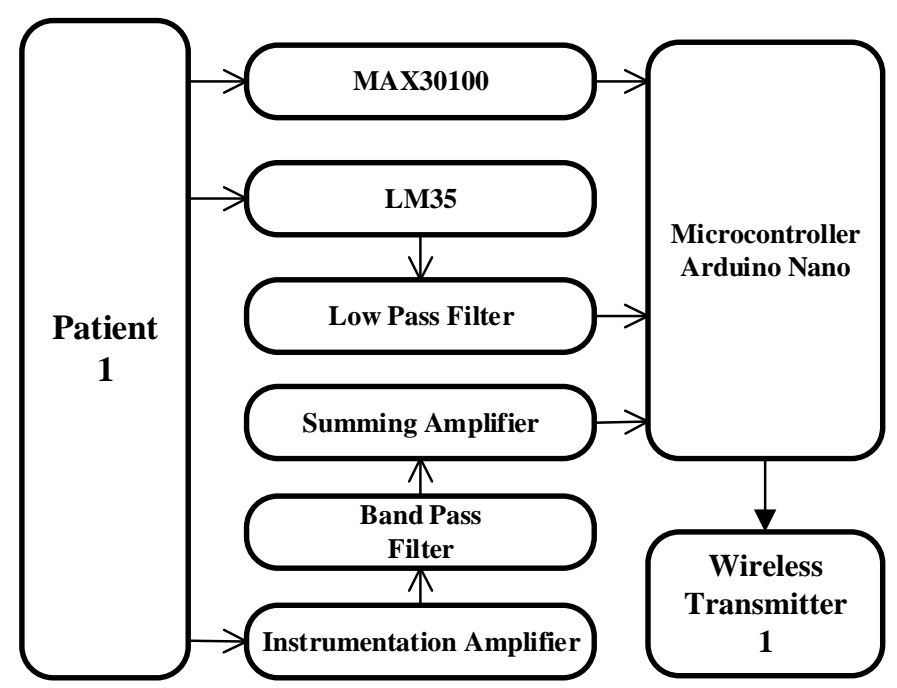

Fig. 1. The diagram block of transmitter 1 .

\section{THE DIAGRAM BLOCK}

This following is a block diagram of a system module consisting of block module diagram transmitter 1, block module diagram transmitter 2 and block module receiver. The MAX30100 sensor will detect SPO2 levels in the blood of the patient and convert them in digital form and then be processed in a microcontroller. The LM 35 sensor will detect the temperature of the patient's body and the analog values obtained will be processed by a microcontroller and converted into a digital value. These sensors will be sent using data encoding so that they do not mix or collide with other data that has been set up in the microcontroller. Digital data sent then received by a personal computer (PC) via Wireless. Then the data is processed with the Delphi program and displayed on the monitor.

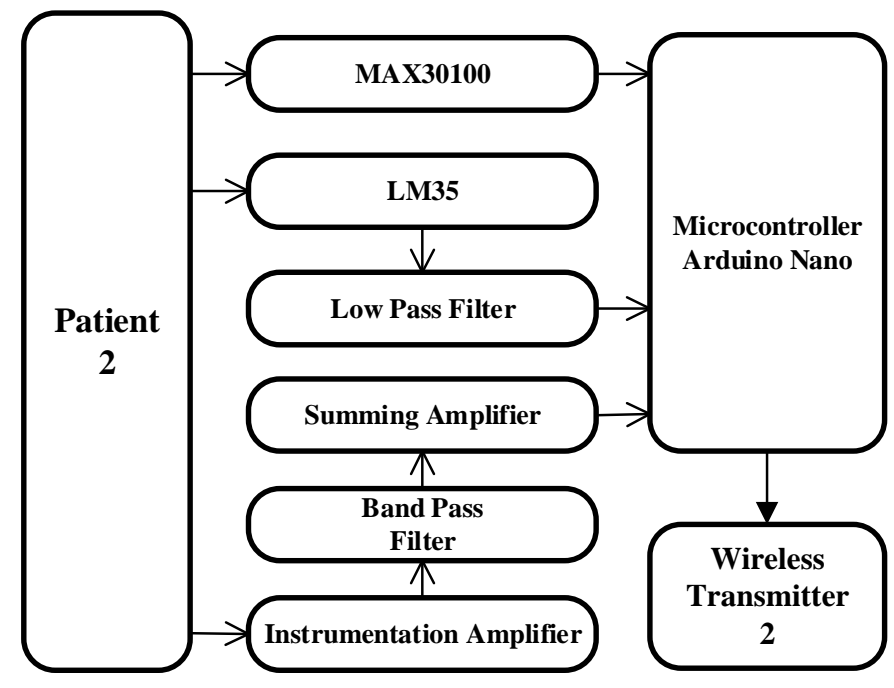

Fig. 2. The diagram block of transmitter 2

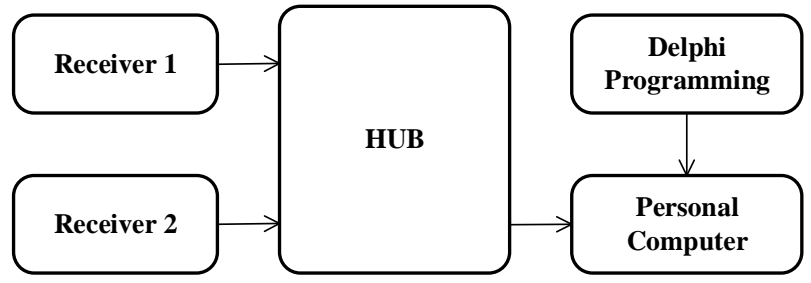

Fig. 3. The diagram block of receiver

\section{E. THE FLOWCHART}

In the flowchart of temperature and $\mathrm{SpO} 2$ transmitter as shown in Fig. 4, after the temperature sensor detects the temperature of the patient's body, the resulting analog value will be processed into digital data (ADC) on the microcontroller and also in spo2 transmitter, the signal that has been captured by the MAX30100 sensor then goes to the Analog pin and is processed in the microcontroller. Then the data will be sent via transmitter. In the flowchart of temperatur and $\mathrm{SpO} 2$ receiver transmitter as shown in Fig. 5, the user connects / starts the PC to call input data from the receiver. After connecting to a $\mathrm{PC}$, it will initialize the software. Data received from the receiver is then processed on a PC using the Delphi application. Data will then be displayed on the display in the form of digital numbers. 


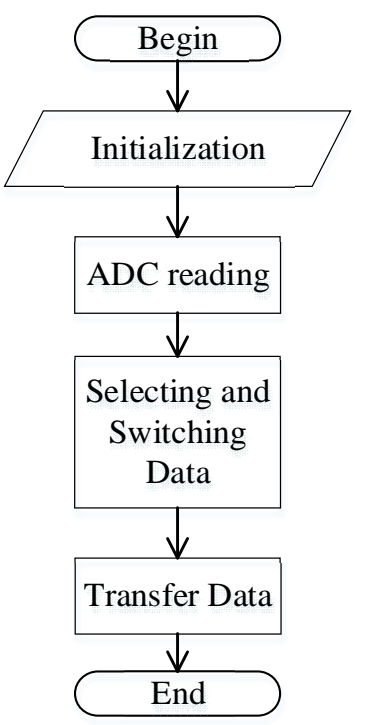

(a)

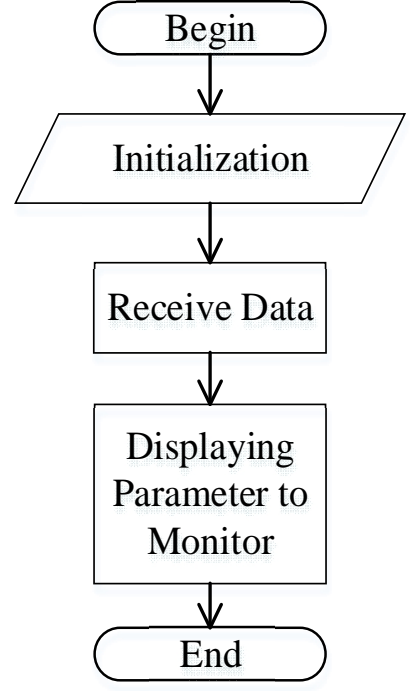

(b)
Fig. 4. The flowchart of termperatur\&SpO2 (a) transmitter and (b) receiver

\section{F. CIRCUIT}

Based on the Fig. 5 and Fig. 6, when the device is turned on, all the circuits are functioning. When the finger is placed on the max30100 sensor, the sensor begins to read the oxygen saturation value in the blood and the data will be processed in Arduino which enters via the analog pin. The LM35 sensor will also read body temperature. The output from the LM35 will go to the Arduino ADC pin and processed to get accurate results. All data that has been processed will be sent using the Xbee wireless transmitter which will later be received by the Xbee receiver and displayed on a PC using the Delphi application.

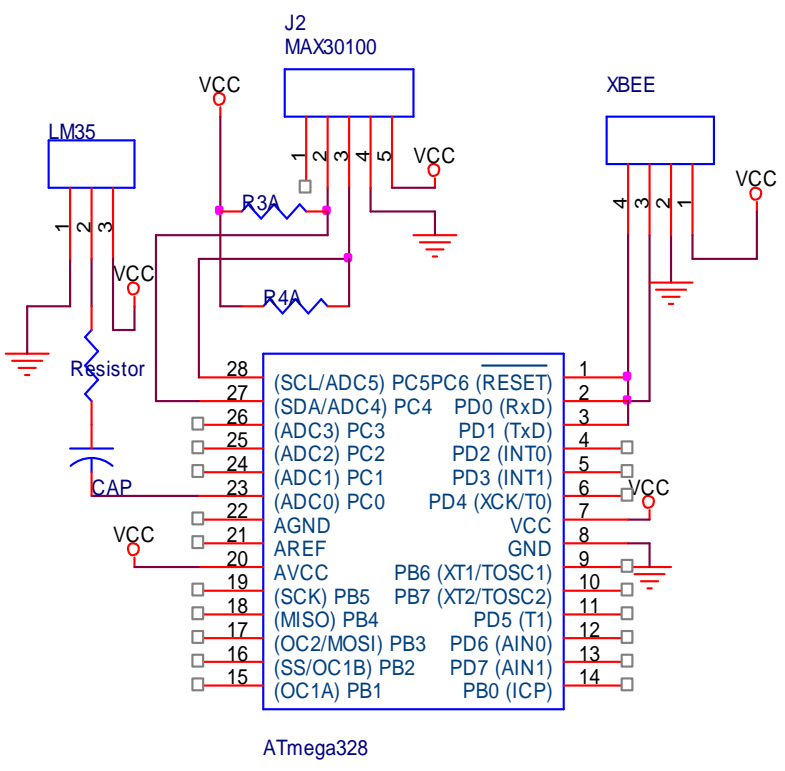

Fig. 5. Minimum system ATMega328
The Transmitter block functions as a sender of data that has been processed in Arduino to the receiver. Xbee is connected to the minimum system (Arduino Uno) contained in each module. The following is a series of transmitter blocks:

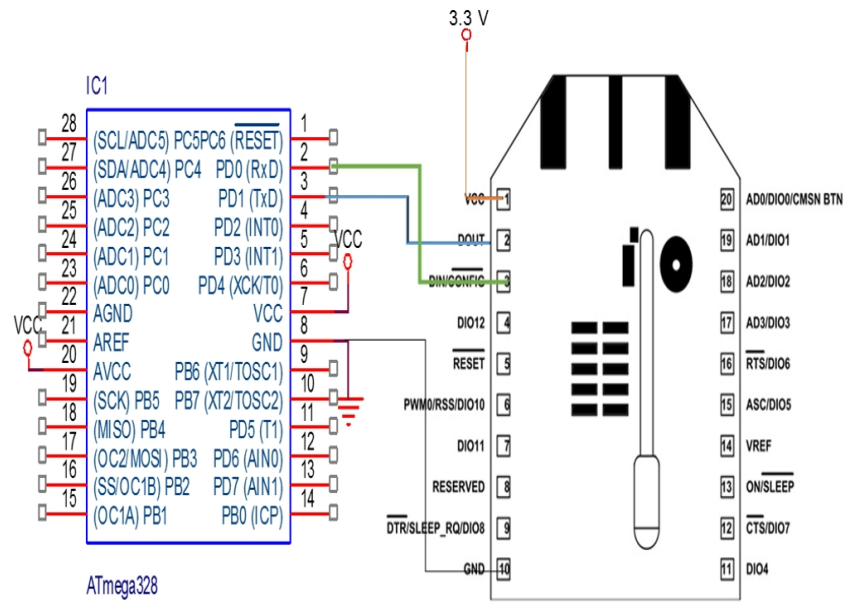

Fig. 6. Wireless Module Transmitter

The receiver block functions as a receiver of data sent by the transmitter block. 2 data transmitters will be received by each receiver then connected to a PC via USB HUB. Data that enters the PC will be displayed using the Delphi application. Here are the receiver blocks (Fig. 7):

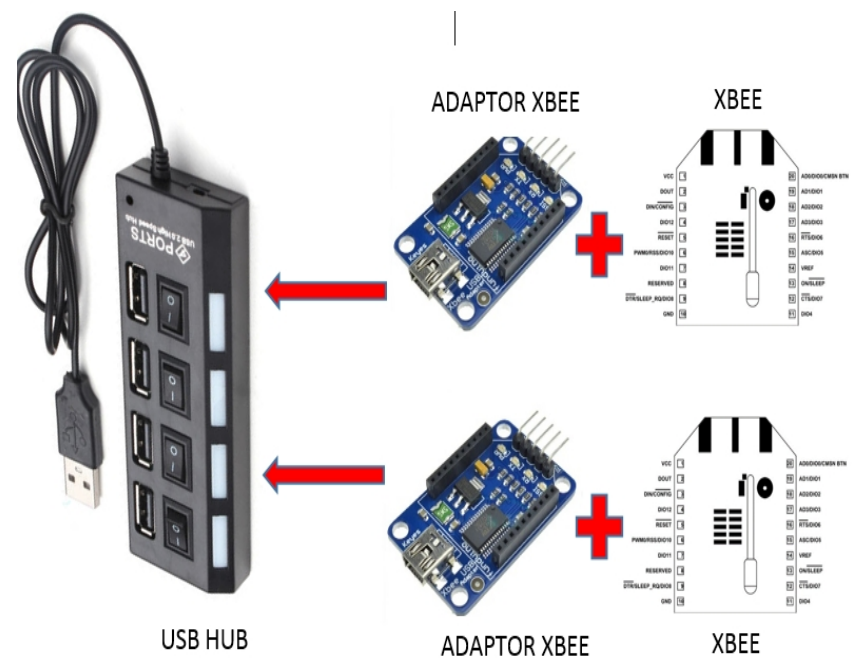

Fig. 7. Wireless Module Receiver

\section{RESULTS}

In this study, the MAX30100 sensor for $\mathrm{SpO} 2$ patient has been tested by an Finger Pulse Oximeter with merk Elitech Technovision type of FOX 1 and the LM35 waterproof sensor for body temperature has ben tested by an Digital Thermometer with merk Magic Star type of MS-202. The output signal from each sensor will sent to PC using a Xbee wireless module. 


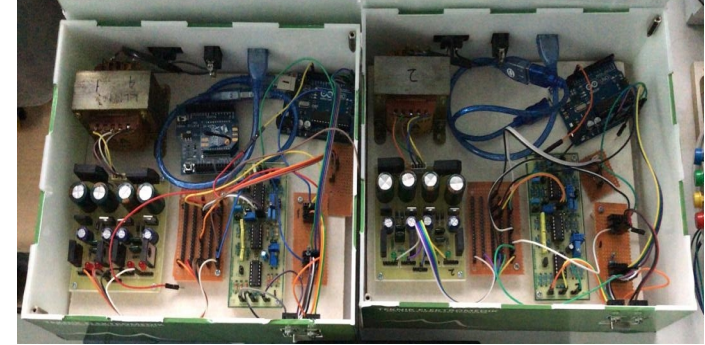

Fig. 8. The Module 1 and 2 design

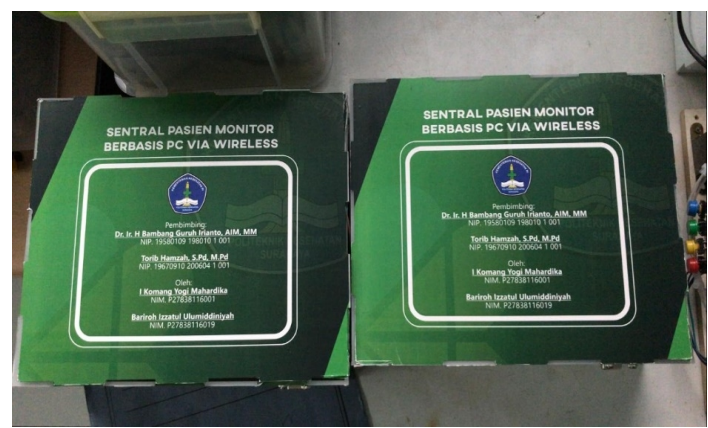

Fig. 9. Display module 1 and 2 from top
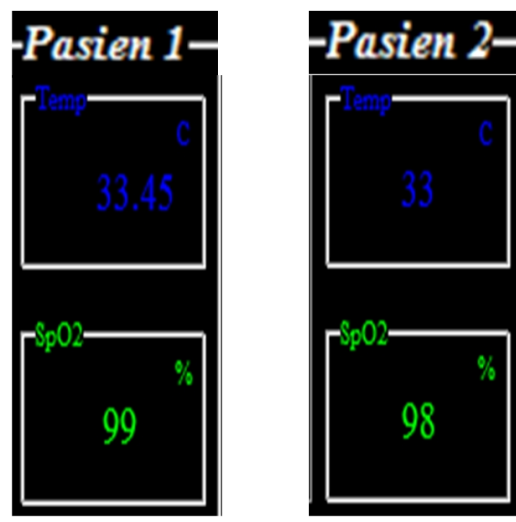

Fig. 10. The display of Central Monitor with distance of 8 Meters
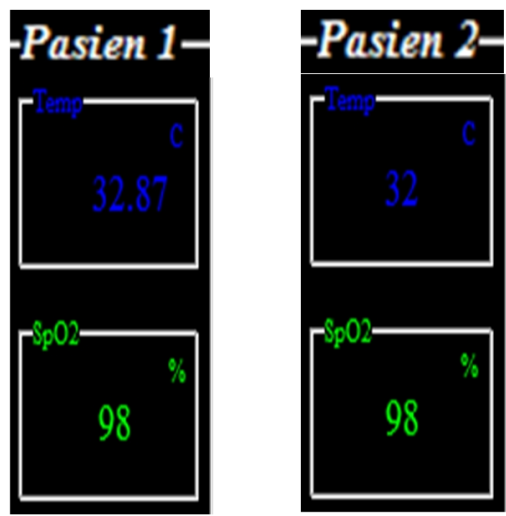

Fig. 11. The display of Central Monitor with distance of 10 Meters
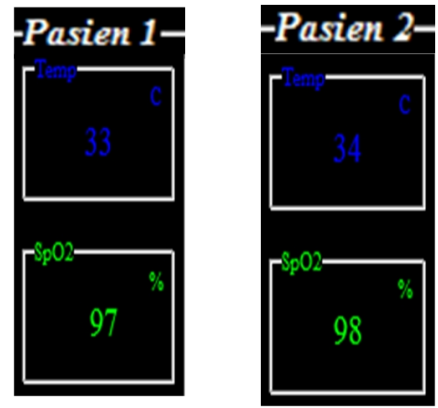

Fig. 12. The display of Central Monitor with distance of 25 Meters
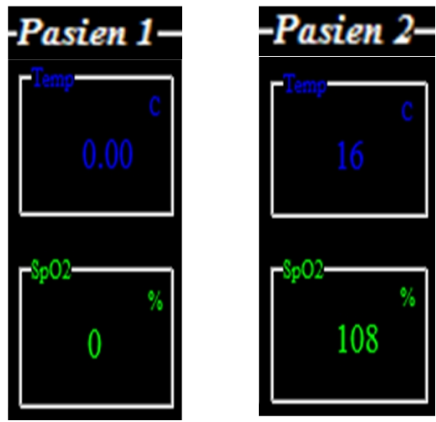

Fig. 13. The display of Central Monitor with distance of 30 Meters

The image of the component part of the central monitor is shown in Fig 9. There is an Arduino Uno microcontroller that functions as an MAX30100 and LM35 data processor, the Xbee Pro wireless module functions to communicate serial data between modules and computers. In this study, module 1 and module 2 transmit data via Wireless, then the data is received by each receiver. Module one and module two will be displayed on the computer in real time and continue. The image of Central Monitoring from module patient monitors one and module patient monitor 2 in the personal computer are shown in Fig. 10, Fig 11, Fig. 12, and Fig. 13. There are 2 parameters (TEMP, SpO2) from each module patient monitor (Fig. 14 and Fig. 15).

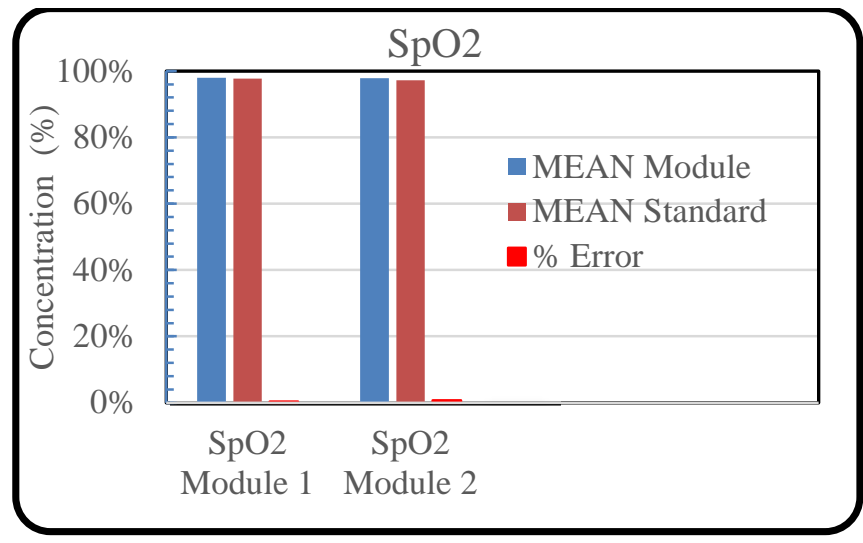

Fig. 14. The Data of SpO2 Module with Standard Device 


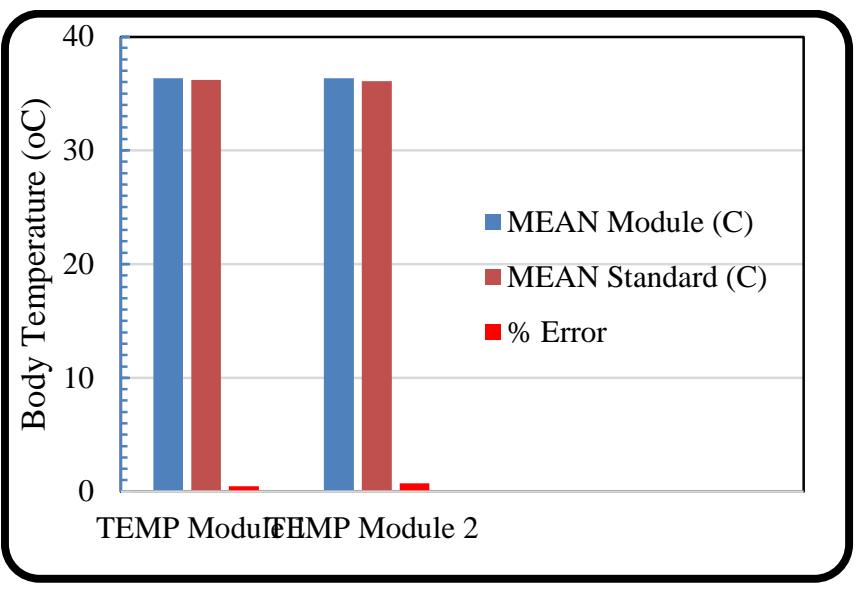

Fig. 15. The Data of Body TEMP Module with Standard Device

\section{DISCUSSION}

After getting the results, the spo 2 data parameters and body temperature parameters were compared with the standard equipment approved. The spo 2 parameter error value obtained in module 1 was $0.1 \%$ and in module 2 it was $0.3 \%$, and the temperature parameter error value was obtained in module 1 it is $0.3 \%$ and in module 2 it is $0.4 \%$. Then, based on Fig. 11, Fig. 12, Fig, 13, and Fig. 14, the test results of the data from module 1 and module 2 are transmitted in real time with distance of 8,10 and 25 meters with a wall barrier. The condition of body temperature and SPO2 are in stable condition, there is no data loss and the viewer application is still stable or not lagging. However, the results of central monitoring began to show problems when the distance was about 30 meters, the display parameters on personal computers began to be unstable / random. The condition of parameters shows random numbers. This is caused by wall barriers that causes the Xbee Pro's wireless power output to be reduced so that the transmission distance traveled is short. This study is a significant improvement, because in the previous study which entitled Central Monitors Based On Personal Computers Using a Single Wireless Receiver (SPO2 Parameter) using HC11 as a wireless, the results of testing the delivery time by distance comparison, it can be concluded that sending using Bluetooth HC-11 can send data as far as 10 meters, and the monitoring display modules 1 and module 2 on the personal computer's centralized screen are carried out alternately (not continue), this is because it only uses 1 data receiver. In practical system, this device allows the centralization process of patient monitor that initially still uses cable, can be replaced with a wireless system that has an easier installation, with a longer transmission distance, more complete parameters and monitoring in real time and continue. So that it will facilitate the performance of the medical staff. Despite the increase, this study is still classified as having incomplete parameters in accordance with standard patient monitor equipment, and the monitoring distance is still relatively close.

\section{CONCLUSION}

The purpose of this study is to make a central monitor tool with monitoring in real time and continue, more complete parameters, via Wireless with greater distance than previous studies. This study shows that a central monitor system that is carried out wirelessly, monitoring will have a long transmission distance if the wireless module has a greater output power, as well as a barrier in transmitting data such as walls, will decrease its output power so that the data transmission distance will be closer and then, in order to be able to be done in realtime and be continued, it needs the same amount of recipient data as the amount of sending data. In summary, this study describes a centralized patient monitoring system that was successfully carried out using Xbee Pro technology and data that was successfully collected on a central monitor without any trouble. Successfully measured 2 physiological parameters namely SPO2 and TEMP each from 2 patient monitor modules. This research shows the monitor system is carried out in real time and continued with a distance of 25 meters, longer than the previous studies. Further experimental requests are needed to increase the number of parameters on the patient monitor according to a standard patient monitor, using a wireless module that can send data longer, making the system portable so it is easy to use.

\section{REFERENCES}

[1] P. S. Purnima, "Zigbee and GSM based patient health monitoring system," 2014 Int. Conf. Electron. Commun. Syst. ICECS 2014, 2014.

[2] D. Hai, P. M. Hung, L. H. P. Trung, D. V. Hung, N. D. Thuan, and P. Dang Hung, "Design of software for wireless central patient monitoring system," Proc. KICS-IEEE Int. Conf. Inf. Commun. with Samsung LTE 5G Spec. Work. ICIC 2017, pp. 214-217, 2017.

[3] S. Sharma and R. Vashisth, "Zigbee Based Centralised Patient Monitoring System," Proc. - 2015 Int. Conf. Comput. Intell. Commun. Networks, CICN 2015, pp. 212-217, 2016.

[4] V. Khedekar, S. Mahajan, and A. Karangale, "A Review on XBEE Technology,” Int. J. Emerg. Technol. Eng. Res., vol. 4, no. 4, pp. 99-101, 2016.

[5] S. Sali and C. S. Parvathi, "Integrated wireless instrument for heart rate and body temperature measurement," 2017 2nd Int. Conf. Converg. Technol. I2CT 2017, vol. 2017-Janua, pp. 457-463, 2017.

[6] Poornendu K, Manoj G, and Kannan E P, "Data Acquisition and Controlling in Thermal Power Plants using a Wireless Sensor Network and LabVIEW," Int. J. Eng. Res., vol. V4, no. 07, pp. 568-572, 2015.

[7] T. Sumalan, E. Lupu, R. Arsinte, and E. Onaca, "Low-cost wireless system for patient monitoring," 2015 E-Health Bioeng. Conf. EHB 2015, pp. 2-5, 2016.

[8] J. Wan, Y. Zou, Y. Li, and J. Wang, "Reflective type blood oxygen saturation detection system based on MAX30100," 2017 Int. Conf. Secur. Pattern Anal. Cybern. SPAC 2017, vol. 2018-Janua, no. 4, pp. 615-619, 2018.

[9] S. Chavan, P. Gadekar, M. Patil, S. P. Tondare, and S. P. Gaikwad, "Solar Powered Non-Invasive Pulse Oximeter and Heart Rate Meter," pp. 52795285,2018 .

[10] R. K. C. Billones, M. P. Vicmudo, and E. P. Dadios, "Fuzzy inference system wireless body area network architecture simulation for health monitoring," 8th Int. Conf. Humanoid, Nanotechnology, Inf. Technol. Commun. Control. Environ. Manag. HNICEM 2015, no. 940nm m, 2016.

[11] T. H. Y. Ling and L. J. Wong, "Elderly infrared body temperature telemonitoring system with XBee wireless protocol," Proc. Int. Conf. Sens. Technol. ICST, vol. 2016-March, pp. 529-535, 2016. 
[12] M. U. H. Al Rasyid, B. H. Lee, and A. Sudarsono, "Wireless body area network for monitoring body temperature, heart beat and oxygen in blood," 2015 Int. Semin. Intell. Technol. Its Appl. ISITIA 2015 Proceeding, pp. 95-98, 2015.

[13] U. Gogate and J. W. Bakal, "Smart Healthcare Monitoring System based on Wireless Sensor Networks," Int. Conf. Comput. Anal. Secur. Trends, CAST 2016, pp. 594-599, 2017.

[14] C. Jing, C. Huasen, and J. Yuanyuan, "Wearable medical guardianship system based on ZigBee," 2017.

[15] K. D. Singh and A. K. Joshi, "Cost effective open source wireless body sensor networking through zigBee," Proc. 2017 IEEE Int. Conf. Commun. Signal Process. ICCSP 2017, vol. 2018-January, no. I, pp. 866-869, 2018.

[16] A. Rerkratn and A. Kaewpoonsuk, "ZigBee based wireless temperature monitoring system for shrimp farm," ICCAS 2015 - 2015 15th Int. Conf Control. Autom. Syst. Proc., no. 1CCAS, pp. 428-431, 2015.

[17] A. E. Osorio, E. A. G. Sanchez, G. F. Garcia, S. F. H. MacHuca, and P. S. L. Lozano, "Evaluation of wireless network based on zigbee technology using XBee modules," 2019 IEEE Int. Conf. Eng. Veracruz, ICEV 2019, 2019.

[18] H. Daud, N. F. I. Gulcharan, M. A. M. Azhar, M. Z. Samsudin, N. M. Nor, and T. Ibrahim, "Monitoring heart rate and body temperature using wireless technology (Zigbee)," Appl. Mech. Mater., vol. 490-491, pp. 1565-1574, 2014.

[19] S. Hadiyoso and S. Aulia, "Multipoint to Point EKG Monitoring Berbasis ZigBee," Semin. Nas. Apl. Teknol. Inf. Yogyakarta, vol. 2135, pp. 1907 5022, 2014.

[20] T. H. Y. Ling, L. J. Wong, J. E. H. Tan, and C. K. Lee, "XBee Wireless Blood Pressure Monitoring System with Microsoft Visual Studio Computer Interfacing," Proc. - Int. Conf. Intell. Syst. Model. Simulation, ISMS, vol. 2015-Octob, pp. 5-9, 2015.

[21] M. Noushad, B. Tauheed, S. A. Khan, and M. A. Khan, "Wireless monitoring of temperature and humidity using sensor array," 12th IEEE Int. Conf. Electron. Energy, Environ. Commun. Comput. Control (E3C3), INDICON 2015, 2016.

[22] I. E. Berliandhy, A. Rizal, S. Hadiyoso, and R. Febyarto, "A multiuser vital sign monitoring system using ZigBee wireless sensor network," ICCEREC 2016 - Int. Conf. Control. Electron. Renew. Energy, Commun. 2016, Conf. Proc., pp. 136-140, 2017.

[23] Almadani, Basem, Manaf Bin-Yahya, and Elhadi M. Shakshuki. "EAMBULANCE: real-time integration platform for heterogeneous medical telemetry system." Procedia Computer Science 63 (2015): 400-407.

[24] Yang, Hongli, and Jihong Chai. "The study and design of a wireless ECG monitoring system." Biomedical instrumentation \& technology 46, no. 5 (2012): 395-399. 


\section{APPENDIX}

\section{1) The Listing Program for Arduino}

In this discussion is about the program on Arduino to send data to a computer

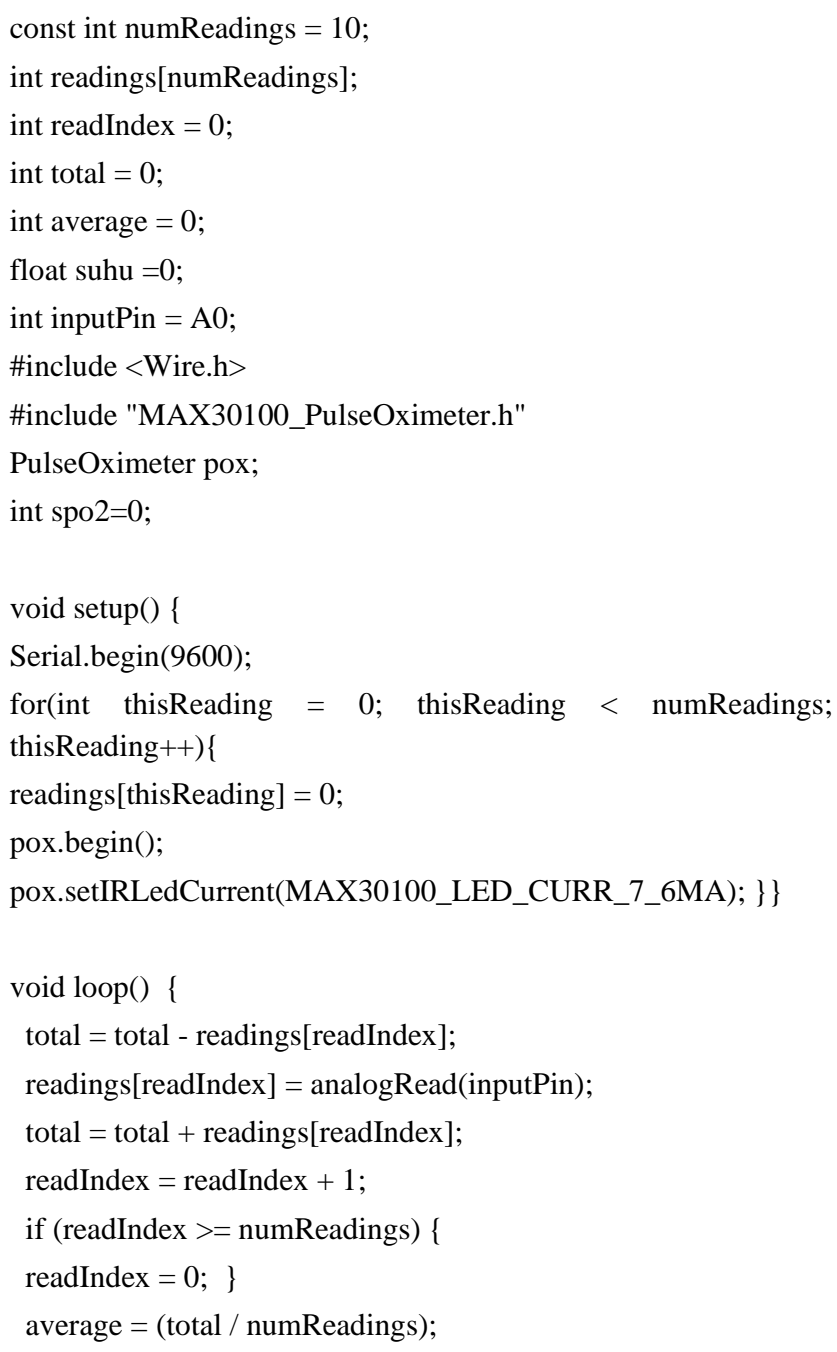

suhu=average $* 3.1$;

suhu=suhu/10.23;

pox.update();

spo2 $=($ pox.getSpO2( $))$;

Serial.print("e");

Serial.print(suhu);

Serial.print("f");

Serial.print("g");

Serial.print(spo2);

Serial.print("h"); \}

\section{2) The Listing Program for Delphi}

In this discussion is about the program on Delphi to displaying data in personal computer

procedure TForm1.Button1Click(Sender: TObject);

begin

ComPort1.ShowSetupDialog;

end;

procedure TForm1.Button2Click(Sender: TObject);

begin

ComPort1.Open;

end;

procedure TForm1.Button3Click(Sender: TObject);

begin

ComPort1.Close;

end;

procedure TForm1.Button4Click(Sender: TObject);

begin

Label3.Caption :='0';

Label5.Caption :='0';

end;

procedure TForm1.ComDataPacket3Packet(Sender: TObject; const Str: String);

begin

Label3.Caption:=Str;

end;

procedure TForm1.ComDataPacket4Packet(Sender: TObject; const Str: String);

begin

Label5.Caption:=Str;

end; 